\title{
EL APRENDIZAJE SOCIAL Y LOS PROFESIONALES DE LA INFORMACIÓN
}

\author{
Sandra Sanz-Martos y Dolors Reig-Hernández
}

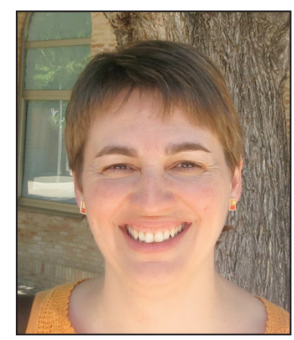

Sandra Sanz-Martos, doctora en sociedad de la información y el conocimiento por la Universitat Oberta de Catalunya (UOC) y licenciada en documentación por la Universidad de Granada, es profesora de los Estudios de Información y Comunicación de la UOC. Directora del posgrado en Redes Sociales e Intercambio de Conocimiento de la UOC y del grupo de investigación eCo (eLearnCenter) donde concentra su investigación en comunidades de práctica y aprendizaje colaborativo en red. http://orcid.org/0000-0003-3028-852X

Universitat Oberta de Catalunya, Estudios de Ciencias de la Información y de la Comunicación Rambla del Poblenou, 156. 08018 Barcelona, España ssanzm@uoc.edu

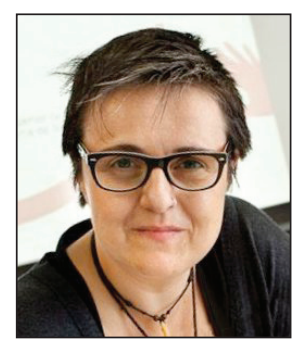

Dolors Reig-Hernández, licenciada en psicología social por la Universidad de Barcelona, posgrado en inserción sociolaboral, master en criminología, master en multimedia, diseño y desarrollo web y editora principal de El caparazón, uno de los espacios más importantes en castellano en su temática. Después de 20 años dirigiendo centros, programas y proyectos de formación, trabaja como freelance, profesora, conferenciante, consultora en empresas, instituciones, administraciones públicas, universidades y escuelas de negocio. Forma parte de comités científicos en social media y educación (Horizon report en educación superior, edición internacional), tendencias de futuro en la web y cultura digital. Asesora a varios gobiernos internacionales. En 2012 publicó "Socionomia" y en 2013 "Los jóvenes en la era de la hiperconectividad". http://orcid.org/0000-0003-2009-8378

Academia El caparazón Banys, 46. 08530 La Garriga (Barcelona), España http://www.dreig.eu/caparazon info@elcaparazon.net

\section{Resumen}

Los medios de comunicación sociales han potenciado la capacidad innata del hombre de compartir conocimiento. Los llamados social media han aumentado lo "social", lo colaborativo y lo cooperativo, revelándose el aprendizaje social como uno de sus principales frutos. Los profesionales de la información, que siempre han sido proclives a compartir información y novedades vinculadas a su disciplina, han sabido aprovechar las posibilidades del aprendizaje social. Reforzados por un sólido networking fomentado durante años por iniciativas como EXIT o E-LIS, los bibliotecarios y documentalistas mejoran su práctica diaria aprendiendo colaborativamente. Mediante las primitivas listas de distribución como IweTel o Públicas, hasta los blogs como Biblogtecarios o Dokumentalistas, cada profesional ha podido ir construyendo su entorno personal de aprendizaje. Se describen varios proyectos de relación profesional en internet y se presta especial atención a la reciente iniciativa Social Biblio y su activa red de aprendizaje.

\section{Palabras clave}

Medios sociales, Aprendizaje social, Entorno personal de aprendizaje, Aprendizaje colaborativo, Redes de aprendizaje, Comunidades de aprendizaje, Comunidades de interés, Comunidades de práctica.

Title: Social learning and the information professionals

\begin{abstract}
Social media have enhanced the innate human capacity for knowledge sharing. The so-called social media have emphasized the 'social' aspect, i.e., the collaborative and cooperative qualities, and social learning is one of the results. Information professionals, who have always been inclined to share information and innovations related to their discipline, have taken advantage of the possibilities that social learning offers. Strengthened by solid networking that has been fostered by years of initiatives like EXIT or E-LIS, librarians and information scientists improve their daily practice by learning collaboratively. From ground-breaking lists like IweTel or Públicas to blogs like Biblogtecarios or Dokumentalistas, each professional has been able to build her or his own personal learning environment. Various professional networking online environments are described, and special attention is given to the recent Social Biblio project and its active learning network.
\end{abstract}




\section{Keywords}

Social media, Social learning, Personal learning environment, Collaborative learning, Learning networks, Learning communities, Communities of interest, Communities of practice, CoP.

Sanz-Martos, Sandra; Reig-Hernández, Dolors (2013). "El aprendizaje social y los profesionales de la información". El profesional de la información, noviembre-diciembre, v. 22, n. 6, pp. 545-553.

http://dx.doi.org/10.3145/epi.2013.nov.07

\section{Introducción}

La tendencia del hombre a aprender de los demás es innata. La historia de la humanidad está repleta de ejemplos de ello. Desde las primitivas evidencias datadas en el Neolítico hasta los gremios artesanales, podemos encontrar muestras de cómo hemos aprendido unos de los otros para poder progresar. A pesar de que la organización industrial propia del taylorismo ha ido primando la especialización y la división individual del trabajo, la eclosión de las redes sociales en todos los ámbitos, también en las organizaciones, parece habernos recordado y ayudado a recuperar esta condición tan natural en el ser humano. Y no sólo eso, sino que además la hemos etiquetado. Los llamados social media nos han aumentado lo "social", lo colaborativo y lo cooperativo y el aprendizaje. Y lo más importante, lo han incorporado como parte integral de la práctica profesional en muchas áreas vinculadas a las tecnologías de la información y la comunicación.

Así, el aprendizaje social es quizás de las mejores cosas que nos ha traído este fenómeno. La colaboración nos permite observar, imitar y cooperar en el proceso de aprendizaje.

Al estilo de los gremios medievales, los profesionales del siglo XXI también aprovechan los medios sociales para aprender los unos de los otros. De forma previsible, podemos encontrar casos pioneros en áreas tecnológicas, como por ejemplo el reconocido caso de los informáticos y programadores implicados en el desarrollo de software libre. Poco después se sumarían otros profesionales como ingenieros o diseñadores gráficos: los primeros compartiendo estrategias para derribar montañas y abrir túneles, los segundos programas y técnicas avanzadas de diseño.

Los profesionales de la información y de la documentación también se cuentan entre los primeros en aprovechar las posibilidades de los medios sociales en su práctica profesional. Han sido tradicionalmente un grupo muy proclive a compartir conocimiento (como no podría ser de otra manera). Un exponente de este interés lo podemos hallar por ejemplo en el uso continuado de las listas de distribución, a diferencia de otras profesiones. Esta predisposición se puede considerar probablemente el caldo de cultivo de lo que ha ido derivando en otras plataformas como blogs o grupos de Facebook, que trataremos más adelante.

\section{Qué es el aprendizaje social}

Vivimos hoy ya "habiendo cruzando el abismo" (Moore, 1991) ${ }^{1}$, conociendo de lleno el fenómeno "Napster" de la cultura en la educación, la educación 2.0, el también denominado open social learning. La cultura del acceso abierto, de la no intermediación, de la formación entre pares, ins- pira los actuales moocs (massive open online courses), entre otras fórmulas, generando la necesidad de evolucionar, de cambiar los modelos de negocio de las instituciones o empresas que quieran seguir siendo competitivas. Sin embargo, estamos hablando de algo con algunos años de recorrido. La UOC ya abordaba el tema en un panel de expertos organizado por la cátedra Unesco de la Universitat Oberta de Catalunya, encargado de analizar el OSL (open social learning) en 2009 (García, 2009). Decíamos entonces (Reig, 2009a): “Emergen nuevos medios y posibilidades, nuevas formas de comunicación, de 'cultura convergente' (Jenkins, 2008) e interactiva, nuevos escenarios y posibilidades de conexión y de socialización a través de lo que hemos denominado el software social, que vacían de sentido el viejo modelo basado en la asunción pasiva, en las aulas cerradas al mundo, en los contenidos producidos por las oligarquías e industrias de la publicación. Son nuevos modelos que apuestan por la apertura, por el fin de los jardines vallados dentro y fuera de la Web. Las redes sociales, preferentemente abiertas, con contenidos distribuidos que el nuevo aprendiz será capaz él mismo de producir, son los ecosistemas esenciales de ese futuro".

La cultura del acceso abierto, de la no intermediación, de la formación entre pares, inspira los actuales moocs

Se trata de un fenómeno que bebe de algunos clásicos, cuyas ideas se trasladan y aumentan de calado en el contexto de uso intensivo de las TIC. Para no extendernos repetimos las enunciadas en el citado panel de expertos:

- conectivismo, la también denominada "teoría del aprendizaje en la era digital", de George Siemens (2010);

- aprendizaje social de John-Seely Brown (2002), que sería pionero en relacionar el fenómeno de la web social con el aprendizaje;

- aprendizaje informal de Jay Cross (2007), que planteó la importancia de este tipo de aprendizaje en empresas, hasta ahora centradas en exceso en lo formal;

- e-learning 2.0: Stephen Downes (2005) y otros orientarían la superación de los gestores de contenido en e-learning para proponer entornos más distribuidos en la web social;

- aprendizaje generativo: Peter Senge (2006) nos habló de organizaciones que aprenden constantemente cuando las orientamos a ello;

- comunidades de práctica: Wenger, White y Smith (2009) ampliaron al contexto virtual la metodología del aprendizaje y la gestión del conocimiento en comunidades; - etc. 


\subsection{Aprender a través de las redes so- ciales y comunidades virtuales}

En clave pedagógica podemos resumir lo que está ocurriendo diciendo que los medios sociales han hecho realidad viejas utopías educativas. Merece la pena, en este sentido, hablar de constructivismo social aumentado, del incremento de oportunidades, la democratización incluso, del acceso al conocimiento que significa la Red. Un concepto muy potente que nos ayudará a entender de qué estamos hablando, es el de la zona de desarrollo próximo de Vygotsky (1978). Se define como la distancia entre el nivel de desarrollo efectivo del alumno (aquello que es capaz de hacer por sí solo) y el nivel de desarrollo potencial (aquello que sería capaz de hacer con la ayuda de un adulto, experto o compañero más capaz): el aumento de su alcance es hoy indudable. En otras palabras, no es nueva la importancia de la conversación con pares y/o expertos en la construcción del propio conocimiento pero sí el alcance global que el tema pueda alcanzar, el gran aumento de esa zona de desarrollo próximo a lo largo de un proceso de aprendizaje.

Sirvan como ejemplo los moocs, especialmente los denominados moocs conectivistas (Reig, 2009b) en los que la construcción social en el aula se amplía a la construcción social con pares, "compañeros" y expertos de todo el mundo. También la metodología pedagógica de los entornos-redes personales de aprendizaje (Reig, 2010) sigue esta lógica, pues la construcción de una red de fuentes de relevancia en Twitter, Facebook, blogs, etc., puede ser un instrumento para la formación permanente y autónoma de valor incalculable. Los PLEs (personal learning environments) son creados por cada persona y están adaptados a su modo de trabajar o a las circunstancias en las que se encuentra en el momento. Cada individuo construye su propio entorno seleccionando los recursos que va a utilizar. Implica cierta apertura a la novedad, curiosidad constante y relación con personas con formaciones diferentes pero intereses comunes.

\subsection{Aprendizaje colaborativo}

Son varias las ventajas de este enfoque pero la principal sería la posibilidad de "aprender a ser" (nos referimos con ello al aprendizaje tácito), aprender a desarrollar la práctica de la profesión participando desde el primer momento en la comunidad de pares. Nos sirve en este sentido el constructo teórico TIC/TAC/TEP (Reig, 2012a; 2012b). Define tres tipos de apropiación:

- TIC o tecnologías de la información y la comunicación. Saber aprovechar la abundancia informativa de Google, las redes sociales en su uso como herramienta de comunicación, etc.

- TAC o tecnologías del aprendizaje y el conocimiento, usar por ejemplo los videotutoriales y recursos multimedia online para el aprendizaje;

- TEP o tecnologías para el empoderamiento y la participación, es una etapa evolutiva que creemos determinante, pues el proceso formativo no estará completo hasta que el estudiante ponga en práctica lo aprendido e incluso genere nuevas ideas o conocimiento en la especialidad en la que se está formando. El aprendizaje abierto, sobre todo en cuanto a su característica social, y el aprendizaje en comunidades sobre el que hablaremos extensamente a continuación, estarían en este nivel o tipo de apropiación avanzada.

\section{Aprendizaje social y profesionales de la información}

Si la primerísima finalidad del profesional de la información es facilitar información al usuario, ésta se cumple incluso entre sus colegas de profesión. El bibliotecario o documentalista comparte lo que sabe dándolo a conocer a través de múltiples plataformas o servicios. Los primeros servicios de internet utilizados por los profesionales de la información fueron las listas de distribución de correo-e. En ellas se comparten novedades, tendencias e incluso preocupaciones. Posteriormente, se ampliaría con un servicio que permite identificar quién es quién en la profesión, con el conocido directorio EXIT. En otro ejercicio de transparencia se pusieron a disposición las publicaciones de los investigadores y expertos a través del repositorio $E$-LIS.

Con la llegada de la web 2.0 proliferaron entre otros productos los blogs, y los grupos de Facebook. Entre estas iniciativas hay que mencionar las comunidades de práctica de la Asociación Española de Documentación e Información (Sedic) (iniciadas en 2008), y los procesos participativos y abiertos impulsados alrededor del think tank Grupo ThinkEPI (Orduña; Baiget; Guallar, 2009). Entre las iniciativas recientes, queremos destacar por su singularidad SocialBiblio, que describiremos y analizaremos luego.

\subsection{Listas de distribución}

En las listas de distribución (también conocidas como foros de debate, listas de correo o listas de discusión) los usuarios de correo electrónico se agrupan por un interés o afinidad común, e intercambian mensajes a través de un servidor de 


\section{Bi $\mathbb{B} \log$ tecarios}

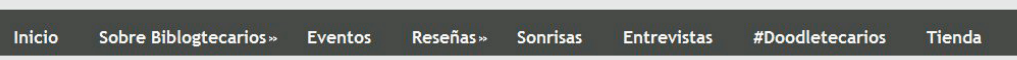

\section{Sobre Biblogtecarios}

¿Qué es BiblogTecarios?

BibliogTecarios es un espacio web colaborativo que busca compartir noticias $y$ opiniones sobre temas de interés para los profesionales del mundo de la Información y la Documentación.

BiblogTecarios es un proyecto que participa de la filosofía del "crowdsourcing": el aprovechamiento de la inteligencia colectiva. De esta manera, en BiblogTecarios podréis encontrar contenidos sobre los diferentes campos de la Biblioteconomía y la Documentación, tratados desde los diferentes puntos de vista de los bloggeros que conforman el proyecto.

\section{¿Cuándo y cómo surgió BiblogTecarios?} BiblogTecarios surge en el año 2010, por iniciativa de Julián Marquina, para dar respuesta a la intención de crear un portal dedicado a noticias v temas de actualidad en http://www.biblogtecarios.es

listas (encargado de distribuirlos a todos los suscriptores) para solventar sus necesidades de información y comunicación sobre un tema determinado. Las listas permiten: comunicarse con cientos o miles de colegas simultáneamente, intercambiar ideas, conocer las actividades y programas desarrollados por otros centros, cooperar, compartir recursos, consultar dudas, estar al tanto de las novedades y establecer vínculos con otros profesionales (Ontalba-Ruipérez, 2009).

Como ya hemos comentado al inicio de este trabajo, los profesionales de la información han mostrado una marcada tendencia hacia el mantenimiento de las listas de distribución, en comparación con otras profesiones. Las primeras datan de principios de los noventa. Las más seguidas en España son Infodoc e IweTel con alrededor de 6.000 suscriptores cada una. Pero existen otras como BibCat con 2.200, Arxiforum con 2.180, Públicas con 1.160, Fidel con 720, Incyt con 295 y un largo etcétera. Hemos seleccionado estas siete -entre un conjunto que supera la treintena en el servidor de RedIRIS- por distintos criterios de significación, algunas por el volumen de suscriptores, otras por reflejar distintos perfiles y temáticas profesionales, sin pretender que ésta sea una muestra estrictamente representativa. A continuación las describimos:

En una CoP no aprenden todos de uno, no habla uno y los otros escuchan, sino que en principio todos tienen algo que aportar

\section{IweTel}

http://www.rediris.es/list/info/iwetel.html

En noviembre de 1993 nació /weTe/ creada por Tomàs Baiget, que buscaba - comenta Ontalba-Ruipérez (2009)- un canal de información profesional más rápido a la hora de comunicar noticias recientes de lo que ya en aquel momento era la revista Information world en español (IWE). Los actuales administradores de IweTel son: Tomàs Baiget (El profesional de la información), Javier Leiva-Aguilera (Catorze.com), Paco López-Hernández (Universidad Carlos III de Madrid) e Isabel Olea (Universidad de León). Desde su fundación, siempre ha sido el principal medio electrónico de comunicación para los profesionales del mundo de la información y la documentación de habla hispana. Algunos de sus temas de debate y discusión son: gestión de contenidos y del conocimiento, biblioteconomía, biblioteca digital, bases de datos y recuperación de información, revistas y comunicación científica, temas laborales, etc.

\section{Infodoc \\ http://www.rediris.es/list/info/infodoc. html}

Lista de la Biblioteca de la Facultad de Traducción y Documentación de la Universidad de Salamanca. Según la propia lista explica, trabajan "con información objetiva, no se trata de una lista de discusión, su vocación es puramente la distribución de mensajes con información profesional del mundo de las bibliotecas y la documentación en general, para que sus suscriptores dispongan de una información actualizada". Está administrada por Julio Alonso-Arévalo, responsable de la Biblioteca. La destacamos por ser una de las listas más importantes en la materia pero no se puede considerar exactamente de aprendizaje.

\section{Arxiforum http://www.rediris.es/list/info/arxiforum.html}

Impulsada y gestionada por la Associació d'Arxivers de Catalunya, Arxiforum se autodefine como el primer foro electrónico del estado español que trata de forma exclusiva la coordinación de recursos, información y opinión sobre la teoría y práctica archivística. Fue creada por Betlem Martínez-Raduà en 1998, quien la moderó hasta 2011.

Fidel (Fuentes de información y documentación en línea) http://www.rediris.es/list/info/fidel.html

Según la propia lista: "el elevado volumen de información disponible en línea gracias a internet retrasa la localización de los recursos más útiles, por ello se hacen necesarios procedimientos como Fidel, que sirvan a la vez de difusores de los recursos de más calidad y de sistema de localización de información".

Su administración corre a cargo de José-Antonio Merlo-Vega, director de las bibliotecas de la Universidad de Salaman$c a$, y está abierta a todos los que deseen difundir recursos, realizar revisiones analíticas o sintéticas de fuentes de información online o solicitar información sobre recursos.

\section{Públicas \\ http://www.rediris.es/list/info/publicas.htmI}

Foro de debate de las bibliotecas públicas. Está dirigida "a personas interesadas en el mundo de la lectura pública y profesionales que trabajan en bibliotecas o instituciones interesadas en el desarrollo de la lectura pública, escuelas, 
facultades y departamentos universitarios de biblioteconomía y documentación, etc. Su objetivo es facilitar el intercambio de información, experiencias y opiniones en torno a las bibliotecas públicas. Tienen cabida noticias, opiniones y peticiones de información y ayuda en torno a temas relacionados con la misión y funcionamiento de la biblioteca pública; legislación en materia bibliotecaria; bibliotecas escolares; cooperación bibliotecaria; tecnologías de la información y de las comunicaciones en las bibliotecas públicas, servicios; formación de usuarios; actividades culturales, cursos, seminarios, congresos, etc.".

Incyt (Indicadores en ciencia y tecnología) http://www.rediris.es/list/info/incyt.html

Creada en 2008, se trata de una lista centrada especialmente en comunicación e indicadores científicos y la práctica bibliométrica. El número de mensajes semanales es muy bajo debido a que sus moderadores (Tomàs Baiget, de El profesional de la información, y Fernanda Peset y Antonia Ferrer-Sapena de la Universidad Politécnica de Valencia, UPV) son estrictos a la hora de aprobarlos, pero hemos querido destacarla porque es la única con un perfil más claramente científico.

\section{BibCat}

http://groups.yahoo.com/group/bibcat

Lista de distribución creada en 1998 con el objetivo generalista de fomentar el intercambio de informaciones, conocimientos y experiencias sobre archivística, biblioteconomía y documentación en lengua catalana.

Como hemos podido observar entre las dos listas más seguidas hay una diferencia. Infodoc, tal y como ellos mismos aclaran no es una lista de discusión, si no simplemente informativa. Este hecho la aleja de ser un posible punto de partida de algo cercano al aprendizaje social. IweTel, en cambio, sí que reúne condiciones para ello puesto que sus seguidores comparten informaciones, experiencias y discuten sobre cuestiones de su interés. Cualquiera puede lanzar una duda y ser resuelta por uno de sus miembros - aunque éste no sea su uso más habitual-. Algo similar sucede con BibCat de corte también generalista. Por el contrario, Arxiforum quizá por ser su centro de interés más acotado reúne las características de lo que más adelante definiremos como una comunidad de interés. De características bien distintas aunque próximas a las de Infodoc es la lista Fidel. En este caso tampoco es la discusión una de las finalidades, si no la identificación o recopilación de fuentes de información de la Red. La filosofía de esta lista responde a la del trabajo colaborativo en red, puesto que solicita "colaboraciones de todas aquellas personas que hayan realizado revisiones de recursos web sobre cualquier disciplina a fin de difundir sus resultados entre los suscriptores de la lista". El enfoque y las características de la lista Públicas son similares a las de Arxiforum probablemente también contribuye a ello la acotación de su centro de interés. Por último está Incyt con un número de seguidores mucho menor que las anteriores pero con un potencial estimulante. Sus miembros profundizan en su práctica investigadora y podría ser el embrión perfecto de otro tipo de comunidad, la de práctica, que también describiremos más adelante.

\subsection{Directorios}

\section{EXIT (Expertos en el tratamiento de la información)} http://www.directorioexit.info

Fundado por Tomàs Baiget y Josep-Manuel Rodríguez-Gairín en 2005, es el directorio de los profesionales de la información. En noviembre de 2013 tiene 3.050 miembros, 62\% españoles. Permite a los profesionales de la biblioteconomía, la documentación y la archivística ponerse en contacto con otros expertos del sector. La inclusión de nuevos miembros está moderada por un comité evaluador internacional que valora de manera flexible los aspectos siguientes entre otros: experiencia profesional de responsabilidad en bibliotecas o centros de información y documentación, participación en proyectos de investigación, actividad docente, publicación de libros y artículos y ser citado en Google Scholar. Sus miembros se comprometen a mantenerlo lo más completo y actualizado posible. La condición innata del hombre a compartir y a aprender los unos de los otros está altamente ligada al sentimiento de pertenencia. Compartimos y aprendemos de nuestros pares, aquellos que son iguales a nosotros y pertenecen a un grupo social similar. El sentimiento de pertenencia se alimenta en lugares como EXIT.

\subsection{Repositorios}

\section{E-LIS (Eprints in library and information science) http://eprints.rclis.org}

Es un repositorio temático especializado en archivística, bibliotecología, comunicación, documentación e información, en funcionamiento desde enero del 2003, y su cobertura es internacional. En noviembre de 2013 tiene depositados 


\section{Info tecarios}

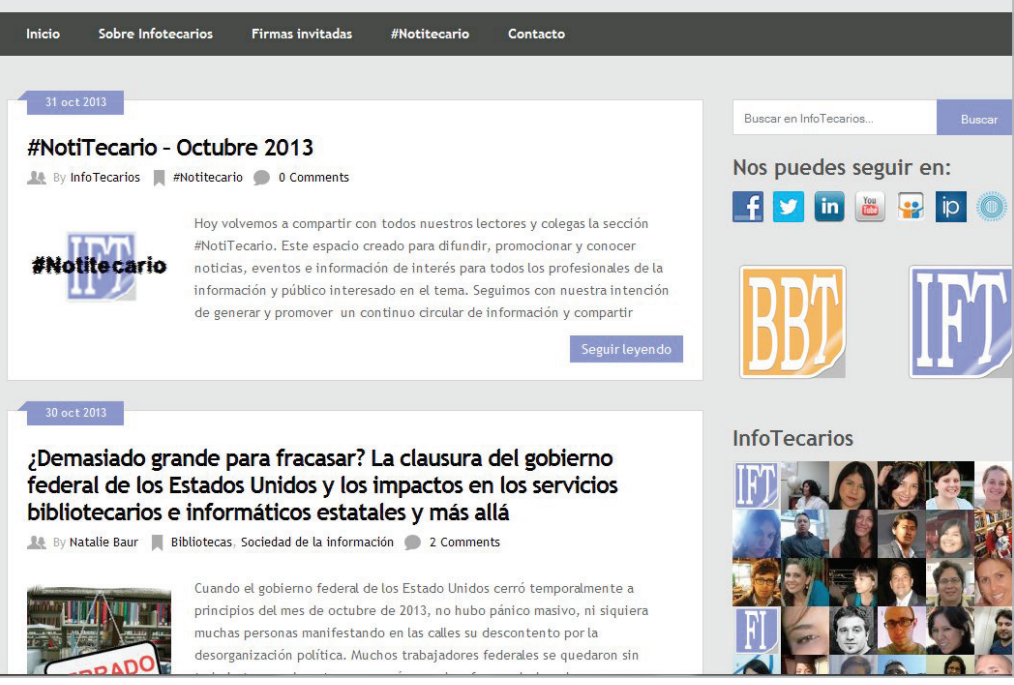

http://www.infotecarios.com contenidos, usos y aplicaciones.

\subsection{Blogs}

Como hemos indicado desde el principio de este artículo, los profesionales de la información han sido pioneros en el intercambio de conocimiento e intereses alrededor de su práctica profesional. No es pues de extrañar la existencia de experiencias pioneras de blogs como Catorze, Deakialli, Trucos de Google, Sedic blog, El documentalista enredado, Bibliotecarios 2.0, Gamoia, Bauen, etc. (Torres-Salinas; Delgado-López-Cózar; Cabezas-Clavijo, 2008).

Algunos de los blogs más populares se han convertido en referentes sobre novedades y tendencias. ¿Cuántos de nosotros hemos aprendido qué es un SEO, un SEM o un content curator a través de Biblogtecarios o Dokumentalistas? Estos blogs son más que fuentes de información, son auténticas fuentes de aprendizaje. Es decir, lo que se denominan entornos persona-

15.600 documentos. E-LIS se basa en el trabajo de voluntarios de todo el mundo y no es comercial (Peset; FerrerSapena; Baiget, 2008). E-LIS aumenta la visibilidad de los trabajos, resulta útil para los bibliotecarios que quieren estar al día de las novedades en su campo y para los autores que tienen la posibilidad de contar con un espacio en donde difundir sus trabajos. Actúa para la comunidad de bibliotecarios y documentalistas no sólo como un recurso donde pueden almacenar, conservar y recuperar documentos a texto completo sobre la disciplina, sino también como una vía de divulgación y una opción para la unión de los profesionales de la información en una comunidad científica en busca de la consolidación de la ciencias de la información y documentación como disciplina científica.

Promueve la filosofía del acceso abierto, facilitando los documentos a todos.

\section{Listas, blogs y grupos de Facebook han acabado formando parte del entorno personal de aprendizaje de los docu- mentalistas, bibliotecarios y archiveros}

El espíritu de E-LIS responde fielmente al resto de iniciativas descritas hasta ahora. El dar a conocer, poner a disposición del resto del gremio, y compartir el conocimiento creado. Los mismos profesionales que comparten — de forma más o menos informal a través de las listas de distribución- información, novedades y experiencias también lo hacen con el conocimiento formal, documentado, lo que garantiza en mayor o menor medida su fiabilidad.

Hasta aquí las principales y originales plataformas del profesional de la información, que surgieron con las primeras listas de distribución y se fortalecieron a través de EXIT y E-LIS. Ahora es el turno de los espacios nacidos de los nuevos medios sociales: los blogs y los grupos de Facebook y sus distintos les de aprendizaje. Veamos las características de algunos:

\section{Biblioblog \\ http://biblioblog.org}

Creado en 2003, a partir de su tercera edición empezó a publicar tanto artículos de opinión de autores y colaboradores, como a ofrecer diferentes opciones de participación. Como indican sus propios impulsores (José-Antonio Merlo-Vega, Natalia Arroyo-Vázquez, Fernando Juárez-Urquijo y Jordi Serrano-Muñoz). Biblioblog pretende "compartir sus visiones, sus opiniones y las informaciones que consideran de interés para la profesión que han elegido, porque también es un blog profesional, el espacio que usamos para destacar recursos, documentos y noticias de interés para nuestro colectivo".

\section{Biblogtecarios \\ http://www.biblogtecarios.es}

Es un espacio web colaborativo que busca compartir noticias y opiniones sobre temas de interés para los profesionales del mundo de la información y la documentación. Es un proyecto que participa de la filosofía del "crowdsourcing": el aprovechamiento de la inteligencia colectiva. De esta manera, en Biblogtecarios se pueden encontrar contenidos sobre los diferentes campos de la biblioteconomía y la documentación, tratados desde los diferentes puntos de vista de los blogueros que conforman el proyecto. Impulsado en 2010 por Julián Marquina-Arenas, se ha convertido en uno de los blogs con más reputación en lengua española. Su vocación es ser un espacio divulgativo y participativo y donde los profesionales del sector puedan estar en constante actualización.

\section{Dokumentalistas \\ http://www.dokumentalistas.com}

Es un espacio colaborativo en red que nace en 2010 con la finalidad de recopilar la gran cantidad de recursos que existen para profesionales de la información y la documentación. Además, es un lugar de encuentro que permite a diferentes profesionales de este ámbito crear y compartir contenidos 
con todos los que se sienten interesados por la documentación y la información. Haciendo uso también de la filosofía del crowdsourcing, Dokumentalistas intenta aportar en unión de sus colaboradores, recursos, reflexiones, noticias, ideas y opiniones, que den una amplia visión, y sean la base para nuevos pensamientos, ideas, iniciativas y líneas de negocio que fomenten el empleo, la innovación, la capacitación profesional y la actividad emprendedora. Sus co-fundadores son Adrián Macías-Alegre y Raquel Tristancho-Casanova. En Dokumentalistas escribe, por ejemplo, Evelio Martínez sobre content curator, cuyos cinco posts son una referencia sobre este tema.

\section{Infotecarios}

http://www.infotecarios.com

Nace en el año 2012 por iniciativa y bajo la dirección de Julián Marquina-Arenas y Diego Ariel-Vega con la intención de aprovechar la experiencia obtenida en Biblogtecarios, tratando de replicar sus objetivos, su funcionamiento y sus características principales conformando un espacio dedicado a brindar noticias, desarrollar ideas y tratar temas de actualidad para el mundo de la información y la documentación basado en un entorno colaborativo, principalmente focalizado en el ámbito latinoamericano.

\subsection{Grupos Facebook}

En los últimos dos años han proliferado los grupos que tratan sobre temas de interés profesional. Son otros entornos personales de aprendizaje. Lugares donde compartir, informarse y aprender de los colegas de profesión. Entre los muchos grupos que existen y los que van surgiendo prácticamente a diario hemos destacado tres:

\section{Software libre para bibliotecas (2.218 miembros) https://www.facebook.com/groups/softwarefree}

Lo acotado de su centro de interés hace que sus aportaciones sean muy pertinentes y útiles. Además cuenta con un alto porcentaje de miembros de prestigio. El grupo comparte novedades, trucos y solución de problemas.

\section{BiblioSEO (1.566 miembros) \\ https://www.facebook.com/groups/188042863611}

Su foco de interés versa en torno a un perfil profesional nuevo en vías de consolidación, que el grupo ayuda a definir y delimitar.

Estos dos ejemplos, por el volumen de miembros y las características de funcionamiento están muy cerca de ser comunidades de interés. En general, los temas de tales comunidades pueden ser tan variados como las aficiones o casuísticas de cada persona, y no tienen por qué estar centrados en la praxis profesional. Aunque compartan técnicas o maneras de hacer, el foco común no gira únicamente entorno al aprendizaje de un aspecto concreto. Otra de sus características distintivas, tal y como indican Armstrong y Hagel (2000), es el mutuo desconocimiento entre sus miembros. Aunque las reuniones presenciales de grupos peque- ños entre miembros pertenecientes a la comunidad son relativamente frecuentes, lo habitual es que un miembro no conozca personalmente a la mayoría de sus compañeros. Incluso es muy probable que no conozca a ninguno de ellos. Son grupos muy numerosos que en muchos casos superan los mil miembros. En las comunidades de interés se comparte información, novedades y, en ocasiones, conocimiento y experiencias.

\section{Amo las bibliotecas! (2.630 miembros) https://www.facebook.com/groups/amolasbibliotecas}

Aunque también hay aportaciones interesantes para la mejora de la práctica diaria, su valor radica en el simple placer de compartir emociones, experiencias o cualquier otra cosa. Y lo más importante, reivindicar lo importantes que son las bibliotecas en nuestras vidas.

\subsection{Social Biblio \\ http://www.socialbiblio.com}

Hemos querido hacer mención especial de esta iniciativa puesto que es probablemente la única que se podría definir como una comunidad o más bien como una red de aprendizaje. Definir en este momento lo primero o lo segundo no importa demasiado - aunque lo haremos más adelante-, lo importante es que la principal finalidad de Social Biblio es el aprendizaje unos de los otros. Y este hecho la convierte en la única iniciativa de estas características. Nació en enero de 2012 de la mano de Paula Traver-Vallés y María GarcíaPuente-Sánchez. Se trata de una propuesta innovadora que ofrece a los profesionales formarse de manera gratuita mediante webinars semanales especializados. Las actividades de Social Biblio se han ido ampliando, adquiriendo también un importante eco en profesionales de Latinoamérica.

Social Biblio "se constituye como una plataforma de formación colaborativa, en la que un día seremos profesores y otro seremos alumnos". Para formar parte de esta "comunidad" sólo hay que suscribirse al blog. Y para colaborar proponer un tema que se domine y se quiera compartir con el resto. Los temas serán aquellos que puedan "ser interesantes para 


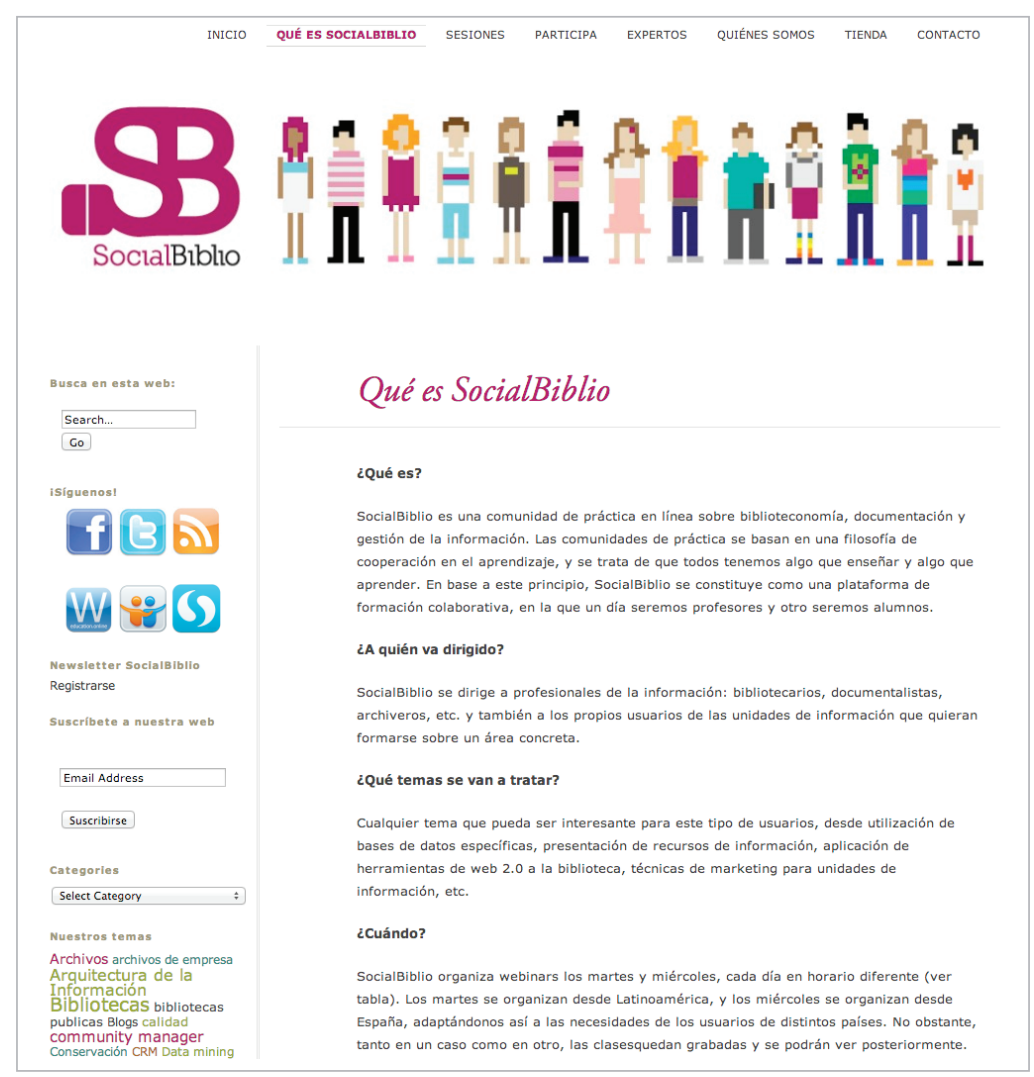

http://www.socialbiblio.com

este tipo de usuarios, desde utilización de bases de datos específicas, presentación de recursos de información, aplicación de herramientas de web 2.0 a la biblioteca, técnicas de marketing para unidades de información, etc.".

Aunque se autodefine como "comunidad de práctica" (CoP), podemos preguntarnos si cumple las características para serlo. Recuperando la definición de CoP de Sanz-Martos (2012) "la comunidad de práctica es un grupo de personas que desempeñan la misma actividad o responsabilidad profesional y que, preocupados por un problema común o movidos por un interés común, profundizan en su conocimiento y pericia en este asunto a través de una interacción continuada".

No hay duda de que se tratan temas de interés, pero para ser una "comunidad de práctica" sus miembros deberían aprender entre todos, compartiendo su conocimiento, experiencia y pericia en cada tema. En una CoP no aprenden todos de uno, no habla uno y los otros escuchan, sino que en principio todos tienen algo que aportar e intercambian conocimientos de igual a igual. Y lo más importante, sobre un mismo centro de interés.

Teniendo en cuenta estas diferencias, podríamos decir que Social Biblio es una "red de aprendizaje colaborativa". Las redes sociales se convierten en redes de aprendizaje cuando se incorporan al ámbito académico y su potencial se usa para fines educativos. Pero sea como fuere Social Biblio sigue siendo una iniciativa brillante, útil y muy valiosa.

\section{Conclusiones}

Desde hace un par de décadas los archiveros, bibliotecarios y documentalistas comparten información, novedades y experiencias a través de un amplio abanico de listas de distribución con un gran número de seguidores en cada una. Esta tendencia, además se ve fortalecida por iniciativas como el directorio EXIT donde los profesionales del sector identifican a sus colegas expertos, o el repositorio E-LIS. De este modo, los suscriptores de IweTel, BibCat o de Públicas reconocen a los participantes de prestigio y valoran en función de éste sus aportaciones.

Obviamente, no todas las listas tienen los mismos objetivos ni funcionan del mismo modo. Algunas son meramente informativas como Infodoc o actúan como recopilador de fuentes como el caso de Fidel. Pero en el caso de IweTel o Públicas la discusión y el debate es una práctica habitual y con ellos se aprende y se comparte conocimiento. A medida que se acota el foco de interés, aprender colaborativamente y profundizar en la práctica profesional deviene una práctica fácil y espontánea. En este sentido tenemos los casos de Arxiforum e Incyt.

Pero este comportamiento natural, que los bibliotecarios, documentalistas y archiveros vienen practicando desde hace años, no se ha quedado aquí, pues gracias a los medios sociales -blogs, grupos de Google, de Yahoo de Facebook...sigue esta imparable (y casi diríamos insaciable) tendencia a compartir del profesional de la información.

\section{El sentimiento de pertenencia se ali- menta en lugares como EXIT}

Destacamos Biblogtecarios (con su versión iberoamericana Infotecarios) y Dokumentalistas como dos iniciativas de prestigio, de obligada consulta para mantenerse al día sobre novedades y tendencias.

Por último, los más recientes grupos de Facebook como Software libre para bibliotecas y BiblioSEO funcionan como comunidades de interés, donde sus miembros comparten informaciones, novedades, trucos y soluciones.

Listas, blogs y grupos de Facebook han acabado formando parte del entorno personal de aprendizaje de los documentalistas, bibliotecarios y archiveros. No cabe duda que la manera de informarse y aprender ha cambiado también en este gremio.

Tanto es así, que en este contexto ha surgido Social Biblio donde los expertos comparten su conocimiento con neófitos en nuevas materias de manera alterna. Actuando como una red de aprendizaje que permite profundizar en nuevos conceptos y compartir el proceso de su asimilación de la mano de aquellos que, a su vez, comparten las mismas inquietudes profesionales.

En un futuro próximo habrá que observar cómo evolucionan estas distintas iniciativas. Probablemente se irán focalizando 
y especializando dando pie al nacimiento de nuevos grupos, en unos casos, o consolidándose en otros, convirtiéndose en comunidades de práctica.

\section{Nota}

1. En su libro Crossing the chasm, Geoffrey A. Moore argumenta que hay un abismo entre los primeros en adoptar un nuevo producto (los entusiastas de la tecnología y visionarios) y el grueso de compradores (los pragmáticos). Moore cree que unos y otros tienen expectativas muy diferentes, y trata de profundizar en ellas y sugerir técnicas para cruzar con éxito el "abismo": elección de un mercado objetivo, comprender el concepto de producto total o completo, posicionamiento del producto, creación de una estrategia de marketing, elección del canal de distribución más adecuado, y precio.

\section{Bibliografía}

Armstrong, Arthur G.; Hagel, John (2000). The real value of online communities. In: Lesser, E. L., Fontaine, M. A. \& Slusher, J. A. Knowledge and communities, pp. 85-98. Boston: Butterworth-Heinemann, 2000.

Baiget, Tomàs; Guallar, Javier (2009). "Networking y comunidades en la web social. Tres proyectos para mejorar la comunicación". En: I Congreso intl de ciberperiodismo y web 2.0, Bilbao, 11-13 noviembre.

http://eprints.rclis.org/13769

Brown, John-Seely; Duguid, Paul (2002). The social life of information. Cambridge: Harvard Business School Press. ISBN: 9781578517084

Cross, Jay (2007). Informal learning: Rediscovering the natural pathways that inspire innovation and performance. California: Pfeiffer. ISBN: 9780787981693.

Downes, Stephen (2005). "E-learning 2.0". elearn magazine. http://elearnmag.acm.org/featured.cfm?aid $=1104968$

García, lolanda, ed. (2009). El open social learning y su potencial de transformación de los contextos de educación superior en España. Informe final. ISBN: 9788469342503

http://openaccess.uoc.edu/webapps/o2/ bitstream/10609/2062/6/informe_OSL_final.pdf

Jenkins, Henry (2008). Convergence culture: La cultura de la convergencia de los medios de comunicación. Barcelona: Ed. Paidós. ISBN: 8449321530

Moore, Geoffrey (1991). Crossing the chasm: Marketing and selling high-tech products to mainstream customers. USA: Harper Business Essentials. ISBN: 0060517123

Ontalba-Ruipérez, José-Antonio (2009). “Análisis de contenido de la lista de correo electrónico IweTel (2001-2007)". El profesional de la información, enero-febrero, v. 18, n. 1, pp. 50-61. http://dx.doi.org/10.1076/epri.11.1.14.9064

Orduña-Malea, Enrique; Guallar, Javier; Baiget, Tomàs (2009). "El Grupo ThinkEPI: un think tank en información y documentación". En: 11 as Jornadas españolas de documen- tación, Zaragoza, 20-22 mayo.

Peset, Fernanda; Ferrer-Sapena, Antonia; Baiget, Tomàs (2008). "Evolución social y networking en la comunidad biblio-documental". El profesional de la información, noviembre-diciembre, v. 17, n. 6, pp. 627-653.

http://dx.doi.org/10.3145/epi.2008.nov.05

Reig-Hernández, Dolors (2009a). Open social learning en España.

http://www.dreig.eu/caparazon/2009/06/30/open-sociallearning-en-espana-primera-presentacion

Reig-Hernández, Dolors (2009b). Moocs: Formación abierta online.

http://www.dreig.eu/caparazon/2008/09/09/moocsformacion-abierta-online-primera-colaboracion-en-labrujula-verde

Reig-Hernández, Dolors (2010). “Entornos profesionales/ personales de aprendizaje en las organizaciones. Propuesta para el programa Compartim". En: Congreso intl EDO 2010: Nuevas estrategias formativas para las organizaciones. Ed. Wolters Kluwer Educación. Joaquín Gairín (ed.). ISBN: 978 8471979971

Reig-Hernández, Dolors (2012a). Socionomía. Barcelona: Ed. Deusto Planeta. ISBN: 9788423409594

Reig-Hernández, Dolors (2012b). Aprendizaje y evolución de lo tecnosocial.

http://www.dreig.eu/caparazon/2012/03/07/aprendizajetecnosocial

Sanz-Martos, Sandra (2012). Comunidades de práctica: el valor de aprender de los pares. Barcelona: Editorial UOC. ISBN: 8490290237

Senge, Peter (2006). La quinta disciplina: el arte y la práctica de la organización abierta al aprendizaje. Buenos Aires: Granica. ISBN: 9506414300

Siemens, George (2010). "Conectivismo: Una teoría de aprendizaje para la era digital". En: Aparici, R. (ed.), Conectados en el ciberespacio. Madrid: Ed. UNED. ISBN: 97884 36261400

Torres-Salinas, Daniel; Cabezas-Clavijo, Álvaro; DelgadoLópez-Cózar, Emilio (2008). "Análisis métrico de los blogs españoles de blioteconomía y documentación (2006-2007)". El profesional de la información, enero-febrero, v. 17, n. 1, pp. 38-48.

http://dx.doi.org/10.3145/epi.2008.ene.04

Vygotsky, Lev S. (1978). Mind in society. The development of higher psychological processes. Massachusetts: Harvard University Press. ISBN: 9780674576292

Wenger, Etienne; White, Nancy; Smith, John D. (2009). Digital habitats. Portland: CPsquare. ISBN: 9780982503607

Xarxa Telemàtica de Catalunya (2013). Pla TAC. http://www.xtec.cat/web/centres/projeducatiu/platac 